\title{
Delay-sensitive and Multimedia Communication in Cognitive Radio Sensor Networks
}

\author{
A. Ozan Bicen ${ }^{\mathrm{a}, *}$, V. Cagri Gungor ${ }^{\mathrm{b}}$, Ozgur B. Akan ${ }^{\mathrm{a}}$ \\ ${ }^{a}$ Next-generation Wireless Communications Laboratory, \\ Department of Electrical and Electronics Engineering, Koc University, Istanbul, 34450, Turkey. \\ ${ }^{b}$ Department of Computer Engineering, Bahcesehir University, Istanbul, 34353, Turkey.
}

\begin{abstract}
Multimedia and delay-sensitive data applications in cognitive radio sensor networks (CRSN) require efficient real-time communication and dynamic spectrum access (DSA) capabilities. This requirement poses emerging problems to be addressed in inherently resource-constrained sensor networks, and needs investigation of CRSN challenges with real-time communication requirements. In this paper, the main design challenges and principles for multimedia and delay-sensitive data transport in CRSN are introduced. The existing transport protocols and algorithms devised for cognitive radio ad hoc networks and wireless sensor networks (WSN) are explored from the perspective of CRSN paradigm. Specifically, the challenges for real-time transport in CRSN are investigated in different spectrum environments of smart grid, e.g., $500 \mathrm{kV}$ substation, main power room and underground network transformer vaults. Open research issues for the realization of energyefficient and real-time transport in CRSN are also presented. Overall, the performance evaluations provide valuable insights about real-time transport in CRSN and guide design decisions and trade-offs for CRSN applications in smart electric power grid.
\end{abstract}

Keywords: Cognitive radio, transport layer, real-time, multimedia transport, delay-sensitive data, rate control, sensor network, smart grid

\section{Introduction}

Dynamic spectrum access (DSA) schemes have been offered to resolve inefficient overall utilization problem of wireless spectrum. Cognitive radio (CR) [1] is the emerging technology to overcome this challenge and provide DSA capability. With the help of CR, vacant bands in the spectrum can be determined and utilized. In this way, CR can communicate over these vacant spectrum bands opportunistically and increase spectrum utilization efficiency.

In wireless spectrum, some of the bands are reserved to licensed users, and these users have priority to communicate over their allocated channels. CR users must detect the arrival of licensed users and vacate the channel. Then, channels, at which licensed user activity is not observed, are identified by spectrum sensing. Channel selection, i.e., spectrum decision, must be made to decide on the channel to continue ongoing communication. Afterwards, CR users should move to this new channel by spectrum handoff. Spectrum sensing, spectrum decision and spectrum handoff operations constitute the cognitive cycle [2], and its application is not limited to licensed bands, e.g., it can also be applied among cognitive radio users while accessing unlicensed bands to increase efficiency and capacity.

In addition to improving overall spectrum utilization, capabilities of CR may help to preserve limited resources of sensor nodes in sensor networks. Sensor nodes containing cognitive radios basically can access

\footnotetext{
*Corresponding Author. Tel. +90212 3382645.

Email addresses: abicen@ku.edu.tr (A. Ozan Bicen), cagri.gungor@bahcesehir.edu.tr (V. Cagri Gungor ), akan@ku.edu.tr (Ozgur B. Akan )
} 
spectrum opportunistically, and hence sensor network can benefit from the advantages of DSA, and this constitutes a new sensor network paradigm, i.e., cognitive radio sensor network (CRSN) [3]. Multimedia and delay-sensitive applications in CRSN are possible with the integration of low-cost hardware and sensor nodes as in wireless multimedia sensor network (WMSN) [4] case.

Figure 1: Multimedia and delay-sensitive data delivering CRSN topology.

Fig. 1 depicts a possible configuration of a CRSN, delivering delay-sensitive multimedia data. Realtime CRSN is interconnected with CR equipped sensor nodes that enable retrieval of video and audio streams, images and delay-sensitive data. Sensor nodes are responsible for timely delivery of multimedia and delay-sensitive data from event region to sink, based on spectrum opportunities. Additionally, control data distribution between sensor nodes is needed to perform proper cognitive cycle operation. Overall, the collaborative operation of CRSN brings significant advantages over traditional sensor networks, including opportunistic channel usage, adaptability to reduce power consumption, dynamic spectrum access [3]. The existing and potential applications of CRSN span a very wide range, including real-time target tracking and surveillance, homeland security, multimedia delivery, and smart grid. However, realization of these currently designed and envisioned applications directly depends on real-time transport capabilities of the deployed CRSN.

Recently, there has been considerable amount of research efforts, which have yielded many promising transport layer protocols for wireless sensor networks (WSN) [4, 5]. The common feature of these protocols is that they mainly address the energy-efficient and reliable data communication requirements of WSN. However, in addition to the energy-efficiency and communication reliability, spectrum-aware communication paradigm emerges with CRSN, and cognitive cycle aware protocol design is needed to take advantage of cognitive radio. Moreover, many real-world CRSN applications, such as multimedia applications, have strict delay bounds and hence mandate timely transport of the event features from the sensor field to the sink node. Real-time communication necessity further amplifies the challenges due to union of cognitive radio and sensor network, and desires careful consideration of communication delays may occur through opportunistic spectrum access in addition to being spectrum-aware. Consequently, the unique features and application requirements of real-time CRSN call for novel transport solutions, and thus, there is an urgent need for understanding the unique challenges of real-time transport in CRSN.

In this paper, the main design challenges and principles for multimedia and delay-sensitive data transport in CRSN are introduced. The existing transport protocols and algorithms devised for cognitive radio ad hoc networks and WSN are explored from the perspective of CRSN paradigm. Specifically, the challenges for real-time transport in CRSN are investigated in different spectrum environments of smart power grid, e.g., $500 \mathrm{kV}$ substation, main power room and underground network transformer vaults. To this end, the wireless channel has been modeled based on experimentally gathered spectrum parameters in different smart power grid environments (shown in our previous study [6]). Through detailed performance evaluations, it is shown that CRSN could be a promising solution to realize delay-sensitive electric utility monitoring and diagnostic systems, with its intelligent processing and DSA capabilities to deal with noise and radio frequency $(\mathrm{RF})$ interference in smart grid environments. In addition, open research issues for the realization of energy-efficient and real-time transport in CRSN are presented. Overall, the performance evaluations provide valuable insights about real-time transport in CRSN and guide design decisions and trade-offs for CRSN applications in smart electric power grid. To the best of our knowledge, this is the first work on the transport layer focusing on real-time event delivery in CRSN and presenting the challenges and performance of delay-sensitive transport in different smart grid environments.

The remainder of the paper is organized as follows. In Section II, real-time communication issues in CRSN, challenges and overview of existing protocols are given. Results of simulation experiments and the effects of cognitive cycle on multimedia and delay-sensitive data delivery in CRSN are presented in Section III. In Section IV, firstly, the key points of results discussed in Section III are outlined to attract attention to emerging challenges, and then, the open research challenges for design of real-time CRSN transport protocols 
are explored. Finally, the paper is concluded in Section V.

\section{Real-Time Transport in Cognitive Radio Sensor Networks}

In order to realize real-time communication in CRSN, a reliable transport mechanism is imperative. Mainly transport layer is responsible from (a) bridging application and network layers, (b) assuring reliable event transport to the sink while taking application specific QoS requirements into consideration, and (c) regulating the sending rate to prevent and avoid congestion. In order to adapt both the unique characteristics of CRSN paradigm and real-time transport requirements, features of transport layer are contingent on significant modifications to obtain efficient real-time communication in sensor networks. Limited resource nature of CRSN nodes bring consequential constraints on the transport layer protocol design [3]. Moreover, opportunistic spectrum access challenge emerges in CRSN, and brings additional link delays and losses as an expected outcome of variations in spectrum availability due to spectrum mobility.

Furthermore, in addition to the metrics for reliable data transport in CRSN, there exist additional metrics due to the unique characteristics of the real-time transport. These metrics can be outlined as delay and jitter, bandwidth requirement, peak signal to noise ratio (PSNR) for multimedia streaming, and frame delivery probability according to the specific QoS demands of real-time CRSN application.

In this section, we investigate the specific CRSN challenges for multimedia and delay-sensitive data delivery and explore the existing transport solutions developed for WSN and cognitive radio networks together.

\subsection{CRSN Challenges for Real-Time Transport}

In real-time CRSN, sensor nodes should deliver gathered event information in a timely manner. Timeliness gains more importance in real-time communication scenarios than reliability to provide successful event detection and tracking in CRSN. On the other hand, in addition to application-specific requirements such as delay bound, jitter, PSNR, etc., sensor nodes should not interfere with licensed users and use their resources efficiently.

Clearly, real-time CRSN imposes unique challenges due to incorporation of DSA functionalities, which are pointed as follows.

\subsubsection{Intermittent Connectivity}

Upon licensed user arrival, sensor nodes have to vacate the channel. This operation requires periodic sensing of the ongoing communication channel for licensed user activity. After detection of licensed user, spectrum handoff should be performed subsequently. As a result, communication path to sink is continuously disrupted. Additionally, due to operating channel change, spectrum characteristics also change. This brings new loss rate and delay durations on the network. Therefore, PSNR varies due to changing spectrum characteristics. Furthermore, round-trip time (RTT) estimation gets complicated in behalf of varying jitter.

\subsubsection{Licensed User Interference}

Sensor nodes sequentially sense channel for licensed user activity and deliver their time-critic data to the sink in an hop-by-hop manner. Sequential spectrum sensing and data transmission inherently results in miss detection possibility of licensed users. Licensed user activity can occur at the event delivery duration, which will change the loss rate in the channel as miss-detection occurs. Miss-detected licensed users, if still communicating, can be detected in next spectrum sensing period, and with the detection of licensed user spectrum decision and spectrum handoff sequence will occur subsequently, i.e., cognitive cycle takes place. Consequently, successful frame delivery probability and PSNR varies, and due to erroneous received packets energy is wasted. 


\subsubsection{Spectrum Sensing}

Detection of licensed user activities are performed via spectrum sensing functionality of cognitive cycle. Spectrum sensing durations prevent sensor nodes to deliver information to sink since existing radio modules cannot provide sensing and transmitting functionalities at the same time. On the other hand, based on interference limitations with licensed users and noise level of operating channel, spectrum sensing duration may vary [7]. Moreover, heterogeneous spectrum sensing durations can be seen throughout sensor network due to observed heterogeneous licensed user activity in the network. Heterogeneous sensing durations will bring synchronization problems on the path from source to sink. On the other hand, nodes operating at relatively less spectrum sensing duration will have greater ratio of channel access duration to spectrum sensing period, and this will cause in-network asymmetries in utilization of available bandwidth based on rate control algorithm running. Resultant effects will be seen in jitter and delay parameters. Additionally, fairness of bandwidth share between sensor nodes will depend on spectrum sensing duration based on heterogeneous licensed user activity and rate control algorithm.

\subsubsection{Opportunistic Spectrum Access}

Sensor nodes are allowed to communicate only if they find a vacant channel. This means that they have to wait until finding an opportunity to access to a licensed user free channel. Blackouts can occur due to consecutive licensed user arrivals on different channels. This causes abnormal variations in RTT. Moreover, transmitted information may be obsolete by the time it reaches the sink, due to loss of timeliness.

\subsubsection{Spectrum Mobility}

Moving from one channel to another (spectrum handoff) due to detection of licensed user means change of spectrum parameters and also additional delay due to sensing in search of a vacant channel. This causes to fill buffer of sensor nodes with new generated packets and incoming packets from other nodes due to ad-hoc nature. Additionally, variations in the bandwidth and noise levels, and henceforth network capacity is anticipated while moving from one channel to another. Changing spectrum parameters will affect delay, jitter and PSNR values. Previously measured channel conditions will be invalid in new operating channel.

\subsubsection{Spectrum Coordination}

Distribution of control data is essential to keep network synchronized in terms of channel selection and spectrum sensing durations. We assume every sensor node has a single transceiver, therefore, they can only communicate at a single channel at any given time. Thus, spectrum decision and handoff functionalities should be performed with coordination. Moreover, nodes participating in the communication from source to sink should be aware of spectrum sensing durations of the other nodes in the path. This is required to establish a path to the sink. As stated before spectrum sensing nodes cannot communicate with other nodes. This fact brings up energy consumption efficiency due to loss of packets and additional delay due to coordination duration.

\subsection{Overview of Existing Approaches for Real-Time Transport in Sensor Networks}

Recently, there has been many research efforts, which have yielded many promising transport protocols for wireless sensor networks (WSN) [5]. Existing transport protocols are classified in Table 1 according to their scope, packet flow, cognitive radio and real-time communication support, respectively. Albeit providing energy-efficient reliable event delivery functionalities as well as congestion detection mechanisms, none of the existing transport protocols considers both challenges of DSA and constraints of sensor network.

Congestion Detection and Avoidance (CODA) protocol [8] aims preventing possible congestion to keep energy-efficient operation by using hop-by-hop congestion detection algorithm based queue length of intermediate nodes on source-to-sink path, and hence, source node can regulate its rate with a incurred additional delay in congestion control. Event-to-Sink Reliable Transport (ESRT) [9] and Real-Time and Reliable Transport $\left((\mathrm{RT})^{2}\right)[10]$ protocols provide event-based reliability by exploiting spatio-temporal correlation of generated packets by source nodes, and are equipped with congestion avoidance, which performs 
Table 1: Summary of Existing Transport Protocols

\begin{tabular}{|l|l|l|l|l|}
\hline Protocol & Scope & Flow & DSA & Real-Time \\
\hline$C O D A[8]$ & WSN & Up & - & - \\
$E S R T[9]$ & WSN & Up & - & - \\
$P S F Q[1]]$ & WSN & Down & - & - \\
$(R T)^{2}[10]$ & WSN & Up & - & + \\
$R M S T[12]$ & WSN & Up & - & - \\
$T P-C R A H N[13]$ & CRAHN & Both & + & - \\
\hline
\end{tabular}

Figure 2: Two-state Markov chain for licensed user activity model.

based on previous decision interval and control is centralized at sink. Additionally, (RT) ${ }^{2}$ support delaybounded reliability. On the other hand, Pump Slowly Fetch Quickly (PSFQ) [11] address sink-to-sensor transport reliability, e.g, aims to deliver packets from sink to sensor nodes in the network reliably, and in order to retrieve missing packets uses hop-by-hop retransmissions. Reliable Multi-Segment Transport (RMST) protocol [12] provides reliable communication on sensor-to-sink path, supports negative acknowledgement (NACK) oriented $100 \%$ reliability like PSFQ, and both of them point out the importance of hop-by-hop retransmissions of lost packets.

Recently, a TCP enhancement for CRAHN, Transport Protocol for Cognitive Radio Ad-Hoc Networks (TP-CRAHN) [13], which is aims to address challenges of DSA in cognitive radio ad hoc networks, is proposed. Even though its algorithm incorporates spectrum sensing and handoff functionalities, it occupies TCP's window-based congestion control mechanism, which has clearly been shown to have very poor performance in sensor networks [5].

With the unique characteristics and emerging challenges of real-time transport in CRSN is far beyond the scope of existing protocols, i.e., they are not designed with the consideration of real-time communication in CRSN. Packets are usually consist of non-redundant data, e.g., carried information is highly compressed. Therefore, providing end-to-end congestion control acquire significance in order to satisfy real-time multimedia streaming and high data rate delay-sensitive applications' QoS requirements in CRSN. Since equation based rate control is a broadly well-known rate control mechanism for streaming [14-17], and thus, in order to assess the characteristics of transport layer in CRSN, we prefer to employ an equation-based rate control algorithm, e.g., TCP-Friendly Rate Control (TFRC) algorithm [14], which uses the following formula to determine throughput in packets per second as a function of packet loss rate and round-trip time:

$$
T=\frac{1}{R T T\left[\sqrt{\frac{2 p}{3}}+12 \sqrt{\frac{3 p}{8}} p\left(1+32 p^{2}\right)\right]}
$$

where $R T T$ is round-trip time, $p$ is packet loss rate, which is ratio of number of lost packets to transmitted ones. Next, a wide range of simulation experiments are performed to reveal multimedia and delay-sensitive data transport challenges in CRSN.

\section{Multimedia and Delay-Sensitive Data Delivery of Cognitive Radio Sensor Networks in Smart Grids : A Case Study}

In order to reveal real-time CRSN challenges for delay-sensitive and multimedia communication, extensive simulations are studied in two parts, namely delay-sensitive data transport and real-time video streaming in CRSN. Before moving discussion of simulation results, first we explain details of CRSN network architecture and simulation environment as follows. 
Figure 3: Sensor node activity pattern with time.

Performance of CRSN is closely related to licensed user activity. Therefore, determination and prediction of licensed user activity is an acute necessity. Licensed user arrivals are assumed to be independent, hence, inter-arrivals are modeled with exponential distribution. Therefore, licensed user activity can be modeled as two-state birth-death process with birth rate $\beta$ and death rate $\alpha$ as in Fig. 2 , and moving from one state to another follows Poisson arrival process, which implies that length of ON and OFF periods are exponentially distributed [18]. Licensed user ON state means channel is occupied by licensed user and OFF state means channel is vacant and it can be accessed by cognitive radio users [19, 20]. By the help of assumed licensed user activity model, posteriori probabilities can be estimated using renewal theory [21] as:

$$
P_{O N}=\frac{\beta}{\beta+\alpha} \quad P_{O F F}=\frac{\alpha}{\beta+\alpha}
$$

where $P_{O N}$ is the licensed user being active(ON), e.g., using the channel, probability, and $P_{O F F}$ is the licensed user being inactive(OFF), e.g., not using the channel probability. We assume spectrum sensing mechanism works perfectly, e.g., no miss detections and false alarms occurs. Therefore, licensed user activity detection probabilities are taken as directly equal to licensed user activity probabilities in simulations.

The collaborative and low-cost nature of wireless sensor networks (WSN) brings significant advantages over traditional communication technologies used in today's electric power systems. Recently, WSN has been widely recognized as a promising technology that can enhance various aspects of today's electric power systems, including generation, delivery, and utilization, making them a vital component of the next generation electric power system, the smart grid [6, 22-28]. To realize reliable and delay-sensitive electric utility monitoring and diagnostic systems, CRSN is a promising solution, with its opportunities to deal with radio frequency $(\mathrm{RF})$ interference and varying spectrum parameters due to DSA capability [6].

In order to simulate wireless channel in smart grid environments, we have used the wireless channel model and parameters determined in our previous study via field-test experiments [6]. Specifically, as channel model, log-normal shadowing path loss model, which calculates path loss with respect to formula in Eq. 3, is utilized. In this model, signal to noise ratio $S N R(d)_{d B}$ at a distance $d$ from the transmitter is given by:

$$
S N R(d)_{d B}=P_{t}-P L\left(d_{0}\right)-10 \eta \log _{10}\left(\frac{d}{d_{0}}\right)-X_{\sigma}-P_{n}
$$

where $P_{t}$ is the transmit power in $d B m, P L\left(d_{0}\right)$ is the path loss at a reference distance $d_{0}, \eta$ is the path loss exponent, $X_{\sigma}$ is a zero mean Gaussian random variable with standard deviation $\sigma$, and $P_{n}$ is the noise power in $\mathrm{dBm}$. Experimentally determined log-normal channel parameters for different power system environments are given in Table 2 .

For power grid environment, licensed user activity can be perceived as wireless channel conditions that restricts communication of sensor nodes. Although, along the paper licensed user term is used, these results can be applied to challenged spectrum environments of power grid.

We have performed simulations by extending the ns-3 network simulator [29] to enable dynamic spectrum access in sensor networks. 200 nodes and a sink are placed randomly in a $100 \mathrm{~m} \times 100 \mathrm{~m}$ field. 10 source nodes are randomly selected within an event area of radius $15 \mathrm{~m}$, and transmit power $\left(P_{t}\right)$ is set to $10 \mathrm{dBm}$. Packet size is limited to 100 bytes due to energy efficiency issues stated in [30] and nodes are equipped with a single transmitter/receiver with a CSMA/CA based simple medium access control layer.

Sensor node behavior is modeled with data transmission $\left(\tau_{S}^{d}\right)$, spectrum sensing $\left(\tau_{S}^{s}\right)$ and spectrum handoff $\left(\tau_{S}^{h}\right)$ periods as shown in Fig. 3. TFRC [14] is used as transport protocol in the simulation experiments. 10 channels are created for each power grid spectrum environment, e.g., 500kV substation, main power room and underground network transformer vault, given in Table 2. Lastly, each simulation configuration is run 10 times and results are averaged. 
Table 2: Log-normal shadowing path loss model parameters for different power grid environments

\begin{tabular}{|l|l|l|l|}
\hline $\begin{array}{l}\text { Power System } \\
\text { Environment }\end{array}$ & $\begin{array}{l}\text { Path Loss } \\
(\eta)\end{array}$ & $\begin{array}{l}\text { Shadowing } \\
\text { Deviation }(\sigma)\end{array}$ & $\begin{array}{l}\text { Noise } \\
\text { Level }(\mathrm{dBm})\end{array}$ \\
\hline 500kV Substation & 2.42 & 3.12 & -93 \\
\hline Main Power Room & 1.64 & 3.29 & -88 \\
\hline $\begin{array}{l}\text { Underground Network } \\
\text { Transformer Vault }\end{array}$ & 1.45 & 2.45 & -92 \\
\hline
\end{tabular}

In the following sections, to gain more insights regarding the challenges of real-time transport in CRSN, comparative performance evaluations are presented for delay-sensitive data and multimedia transport, respectively.

\subsection{Delay-Sensitive Transport in CRSN}

(a) (b)

(c) (d)

Figure 4: Variation of throughput(a), packet delivery(b), packet receiving frequency(c) and packet delivery ratio(d) with respect to increasing $P_{O N}$ for $\tau_{S}^{d}$ of $1.5 \mathrm{sec}$ and $\tau_{S}^{s}$ of $0.5 \mathrm{sec}$.

Timely delivery of event information is necessary for delay-critical applications in CRSN. To evaluate delay-sensitive transport issues in CRSN, we have performed simulations for different licensed user activity probability and spectrum sensing duration to data transmission duration ratio values in different spectrum environments of a power grid. In order to assess effects of licensed user activity, firstly, we have used constant spectrum sensing duration for all ten channels and changed licensed user state transition rates in Fig. 4 and 5, then, kept licensed user activity constant and changed $\tau_{S}^{s} / \tau_{S}^{d}$ ratio while assessing spectrum sensing duration's effects in Fig. 6 and 7. We have measured average throughput, packet delivery, packet inter-arrival time per second and packet delivery ratio to assess performance.

\subsubsection{Throughput}

Variation of throughput with respect to different licensed user activities and spectrum sensing parameters is given in Fig. 4(a), Fig. 5(a), Fig. 6(a) and Fig. 7(a). In Fig. 4(a), with the increasing licensed user ON probability, different throughput patterns are observed for $\tau_{S}^{s}=0.5 \mathrm{sec}$ and $\tau_{S}^{d}=1.5 \mathrm{sec}$. In $500 \mathrm{kV}$ substation environment, throughput initially increases due to wrong measurement of RTT and packet loss probability. Packet loss probability is measured at the sink for each source and send in acknowledgement feedback packets to the source nodes. Due to its harsh spectrum conditions, in $500 \mathrm{kV}$ substation environment higher path loss exponent is measured, and hence, higher number of packet drops are expected. However, due to fact that acknowledgement packets from sink are not timely received packet loss rate could not being set to its desired value. Moreover, rate is regulated by time-out events in TFRC, however, as RTT gets larger TFRC source waits more for acknowledgement packets to come. Therefore, timely reception of acknowledgement packets is crucial for rate control in CRSN. On the other hand, as $P_{O N}$ keeps increasing, throughput decays for $500 \mathrm{kV}$ substation environment, since RTT becomes dominant factor for controlling throughput. In main power room environment, which has a less path loss exponent than $500 \mathrm{kV}$ substation environment, throughput starts to decrease immediately with the licensed licensed user on probability. RTT becomes dominant factor for throughput calculation in main power room and underground network transformer vault environments. Additionally, throughput slightly increases for underground transformer vault environment while moving 
(a) (b)

(c) (d)

Figure 5: Variation of throughput(a), packet delivery(b), packet receiving frequency(c) and packet delivery ratio(d) with respect to increasing $P_{O N}$ for $\tau_{S}^{d}$ of $1.5 \mathrm{sec}$ and $\tau_{S}^{s}$ of $0.2 \mathrm{sec}$.

(c) $\quad(\mathrm{d})$

Figure 6: Variation of throughput(a), packet delivery(b), packet receiving frequency(c) and packet delivery ratio(d) with respect to decreasing $\tau_{S}^{s} / \tau_{S}^{d}$ ratio, for $\beta=7$ and $\alpha=3$.

(c) (d)

Figure 7: Variation of throughput(a), packet delivery(b), packet receiving frequency(c) and packet delivery ratio(d) with respect to decreasing $\tau_{S}^{s} / \tau_{S}^{d}$ ratio, for $\beta=3$ and $\alpha=7$.

from $P_{O N}=\frac{7}{10}$ to $P_{O N}=\frac{9}{10}$, since rate reductions due to time-outs results in less throughput degradation with respect to degradation due to bursty losses in sensor network.

In Fig. 5(a), throughput stays constant for all licensed user birth-death rates for $\tau_{S}^{s}=0.2 \mathrm{sec}$ and $\tau_{S}^{d}=1.5$ sec. It shows us that as $\tau_{S}^{d}$ gets larger with respect to $\tau_{S}^{s}$, licensed user activity does not effect throughput performance of sensor nodes. As $\tau_{S}^{s}$ is shorter relative to $\tau_{S}^{d}$, spectrum handoff durations do not cause any change in throughput pattern.

As it can be seen from Fig. 4(a), TFRC throughput is not strictly decreasing with increasing licensed user birth rate. However, erroneous measurements of packet losses and RTT cause throughput to take undesirable values. Since injection of more packets cause congestion and leads to packet drops, robust throughput control is essential in real-time CRSN

In Fig. 6(a) and 7(a), variation of throughput is given for different $\tau_{S}^{s} / \tau_{S}^{d}$ ratio while $P_{O N}$ kept constant. In addition to problems in estimation of RTT and p, with the variation of $\tau_{S}^{d}$ congestion problem in sensor network emerges. In Fig. 6(a), it can bee seen for $P_{O N}=\frac{7}{10}$ that decreasing $\tau_{S}^{s} / \tau_{S}^{d}$ ratio results in congestion. For the same packet generation rate, when nodes have more $\tau_{S}^{d}$, more packets are injected into network. Therefore, decreasing $\tau_{S}^{s}$ creates bottlenecks in the network. However, with the further decreasing $\tau_{S}^{s}$, increases in the throughput pattern are observed due to the fact that sensor nodes gain enough $\tau_{S}^{d}$ to deliver delay-critical data to sink. Same pattern for throughput is also seen in Fig. 7(a). However, this time sharper decreases are seen due to $P_{O N}$ is decreased to $\frac{3}{10}$. Since less spectrum handoffs occur because of decreased licensed user activity, more packets are injected into network in simulations of Fig. 7(a), and hence, congestion severity is increased. Additionally, with the decreased $P_{O N}$ in Fig. 7 (a) throughput reaches higher values than in Fig. 6(a).

With the decrease of $\tau_{S}^{s}, \tau_{S}^{d}$ increases relatively, and as a result of this congestion occurs in the network. From this point, increasing $\tau_{S}^{s}$ can be seen as the decrease of the packet injection into network. Therefore, with the decreasing $\tau_{S}^{s}$, we increase the number of packets injected into network, and hence, create bottlenecks and amplify RTT. While keeping to decrease $\tau_{S}^{s}$, these bottlenecks can be diminished as in $500 \mathrm{kV}$ substation environment in Fig. 7(a) while moving from ratio $\frac{1}{4}$ to $\frac{1}{6}$, or bottlenecks can be generated as in main power 
room example in 6 (a) while moving from ratio $\frac{1}{6}$ to $\frac{1}{8}$. The relationship between throughput and $\tau_{S}^{s}$ varies depending on the spectrum environment, licensed user activity pattern and $\tau_{S}^{d}$.

\subsubsection{Packet Delivery}

Packet delivery is given in Fig. 4(b) and Fig. 5(b) for varying $P_{O N}$, while $\tau_{S}^{s}$ and $\tau_{S}^{d}$ are kept constant. In Fig. 6, number of packets delivered to sink strictly decreases even if throughput increases. With the increasing probability of licensed user being $\mathrm{ON}$, spectrum handoffs due to detection of licensed user activity are performed more frequently and exposed delay increases. Additionally, disruption of event delivery by frequent licensed user activity prevents source and intermediate nodes to deliver packets to sink in a timely manner. In Fig. 4(b), although throughput increases while changing $P_{O N}$ from $\frac{3}{10}$ to $\frac{1}{2}$, packet delivery continues decreasing. Inaccurate throughput values can increase the congestion level in the network. Additionally, discrimination of wireless losses and losses due to congestion is required to efficiently control the sending rate of sensor nodes. With its high path loss exponent with respect to other environments, while licensed user ON probability is increased, $500 \mathrm{kV}$ substation environment's throughput decays more drastically than the throughput of other environments even its throughput increases due to wrong determination of RTT and packet loss rate. On the other hand, in Fig. 4(b), for a lower $\tau_{S}^{s} / \tau_{S}^{d}$ ratio, packet delivery stays near constant for all three environments until $P_{O N}$ is increased to $\frac{9}{10}$. With the decreasing $\tau_{S}^{s} / \tau_{S}^{d}$, number of delivered packets at the sink become more resistant to increasing $P_{O N}$, in Fig. 7(b). Although throughput is constant as seen in 7 (b), delivered packets start to decay for $500 \mathrm{kV}$ substation environment, main power room and underground network transformer vault, respectively. Its lossy spectrum conditions make $500 \mathrm{kV}$ substation have less number of packets delivered at the sink with the retransmitted packets. Feedback packets are sent to regulate rate and recover missing packets in case of errors, however, on the average throughput stays constant due inaccurate measured RTT and packet loss rate.

In order to understand effect of $\tau_{S}^{s} / \tau_{S}^{d}$ ratio on packet delivery, simulation results are presented in Fig. 6(b) and $7(\mathrm{~b})$, for $P_{O N}=\frac{7}{10}$ and $P_{O N}=\frac{3}{10}$, respectively. Packet delivery is disrupted by congestion with the decreasing $\tau_{S}^{s} / \tau_{S}^{d}$ ratio due to injection of more packets to the sensor network, in Fig. 6(b) and Fig. 7(b) for underground network transformer vault environment, which has the lowest path loss exponent in all three environments. However, as $\tau_{S}^{d}$ gets larger values than $\tau_{S}^{s}$, delivered packets start to increase due to depleting congestion. This is due to fact that $\tau_{S}^{d}$ becomes dominant over $\tau_{S}^{s}$. Therefore, it is not possible to say packet delivery immediately increase with the decreasing $\tau_{S}^{s} / \tau_{S}^{d}$ ratio, since it is possible to cause congestion with the increasing number of packet injections in densely deployed sensor network. Throughput, i.e., number of packets are being sent, is critical for maximizing packet delivery. Thus, accurate measurement of RTT and packet loss rate once more gains importance. On the other hand, it is certain that decreasing $\tau_{S}^{s} / \tau_{S}^{d}$ ratio sufficiently has a potential improvement impact on packet delivery as it can be seen in Fig. 6(b) for main power room while moving from ratio $\frac{1}{4}$ to $\frac{1}{6}$ and in Fig. 7 (b) for $500 \mathrm{kV}$ substation while moving from ratio $\frac{1}{4}$ to $\frac{1}{8}$, for large and small $P_{O N}$ values, respectively.

Overall, there is a delicate balance between number of injected packets into network and congestion in sensor networks. Hence, received number of packets are depending on congestion, spectrum conditions and cognitive radio parameters such as spectrum sensing and henceforth licensed user activity.

\subsubsection{Average Packet Receiving Interval}

Due to spatial and temporal correlation of the information delivered by sensor nodes [31], it is important to receive required amount of packets in a predefined delay bound in delay-sensitive applications [10]. In Fig. 4(c), 5(c), 6(c) and 6(c) average inter-arrival time of incoming packets at the sink is given in order to assess timeliness of event detection in delay-critical applications. Each of 10 source nodes was set to generate 100bytes packets per $25 \mathrm{msec}$. Therefore, average packet generation inter-arrival by source nodes in the sensor network is considered to be about $2.5 \mathrm{msec}$.

In Fig. 4(c) and 5(c), average inter-arrival time of received packets is strictly increases. Increasing $P_{O N}$ disturbs the delay-sensitive data transport of event. Although sending interval was 0.025 for each of 10 source nodes, e.g., 400 packets are generated per second by all source nodes, sink receives about 250 packets per second, e.g., an average inter-arrival duration of 0.004 sec., for $P_{O N}=\frac{1}{10}$, and $\tau_{S}^{s}$ and $\tau_{S}^{d}$ are equal to 0.5 and $1.5 \mathrm{sec}$, respectively. For $500 \mathrm{kV}$ substation spectrum environment, it increases rapidly up to 0.017 
sec. in Fig. 4, which is undesirable when real-time delivery of event is thought. For a lower $\tau_{S}^{s}$, e.g., 0.2 sec., in Fig. 5(c), average packet inter-arrival also increases sharply with increasing $P_{O N}$, however, it is relatively smaller than the one observed in previous case, e.g., it has a maximum packet inter-arrival duration about $\frac{1}{3}$ of the one in $4(\mathrm{c})$ for all three spectrum environments.

Due to the variation of both throughput and congestion level in the network, average packet receiving interval at the sink does not follow a simple pattern with the decreasing $\tau_{S}^{s} / \tau_{S}^{d}$ in Fig. 6(c) and Fig. 7 (c) for constant $P_{O N}, \frac{7}{10}$ and $\frac{3}{10}$, respectively. For decreasing $\tau_{S}^{s} / \tau_{S}^{d}$ ratio, average packet inter-arrival time follows different patterns. It takes values up to $400 \%$ and $300 \%$ of sending inter-arrival duration, in Fig. 6(c) and Fig. 7(c), respectively. This points out the heavily congested sensor network due to load of 40 packets per source node in a second, and variation of $\tau_{S}^{s} / \tau_{S}^{d}$ can cause this congestion deplete or overwhelm sensor network based on $P_{O N}$. Therefore, consideration of congestion prone densely deployed sensor network's constraints is essential in order to satisfy real-time QoS requirements.

\subsubsection{Packet Delivery Ratio}

In terms of energy efficiency, packet delivery ratio is an important metric for sensor networks with high communication load due to their resource limited nature. We measure packet delivery ratio as the ratio of total received packets by sink to total sent packets by source nodes in the network. Dropped packets in lower layers of source node or in forwarding intermediate nodes and lost packets due to spectrum environment are not received by sink.

In Fig. 4(d), packet delivery ratio decays with the increasing $P_{O N}$. In addition to its lossy propagation environment, frequent spectrum handoffs cause packet delivery ratio in $500 \mathrm{kV}$ substation environment to decay from 0.8 to 0.22 , in Fig. 4(d). In Fig. 5, packet delivery ratio is kept constant (as it was in throughput and packed delivery plots in Fig. 5(a) and in Fig. 5(b), respectively) about 0.7. Packet delivery ratio is about $75 \%$ and $60 \%$ for underground network transformer vault and $500 \mathrm{kV}$ substation, respectively. Which means that, at least one quarter of created packets, together with retransmissions, could not be delivered to the sink due to packet drops or propagation errors. This points out the need for adequate flow control algorithms in CRSN for use with energy limited sensor nodes.

Since lower $\tau_{S}^{s} / \tau_{S}^{d}$ ratio enables source nodes to inject more packets into network to be delivered to sink, congestion spreads from source nodes to intermediate nodes, which forward packets toward sink. In Fig. $7(\mathrm{~d})$, due to low $P_{O N}$ relative to Fig. 6(d), packet delivery ratio does not drop below 70\%. Initially packet delivery decreases, afterwards, it starts to increase since less spectrum handoff delay is exposed due to lower $P_{O N}$, e.g., for main power room environment packet delivery ratio firstly decreases due to congestion and then with the decreasing $\tau_{S}^{s} / \tau_{S}^{d}$ ratio, packet delivery ratio starts to increase in Fig. $7(\mathrm{~d})$. On the other hand, in Fig. 6(d), packet delivery initially decreases while $\tau_{S}^{s} / \tau_{S}^{d}$ ratio decreases, then it turns to be increasing for underground network transformer vault environment.

Based on spectrum environment and communication load on sensor nodes, packet delivery ratio can decrease with decreasing $\tau_{S}^{s} / \tau_{S}^{d}$ ratio. Packet delivery ratio also can be seen as an indicator of energy efficiency, and hence, flow control gains crucial importance in delay-sensitive CRSN applications to both preserve energy and deliver events timely. In order to keep network away from congestion and prevent energy wastage, $\tau_{S}^{s} / \tau_{S}^{d}$ ratio should be taken into consideration deliberately with according $P_{O N}$ and spectrum environment while controlling rate of source nodes.

\subsection{Real-time Video Streaming in CRSN}

In simulations a sample MPEG-4 stream of $10 \mathrm{sec}(30 \mathrm{fps}, 289.58$ Bytes average frame length, $35.39 \mathrm{~dB}$ average frame peak signal-to-noise ratio) is used to assess real-time video streaming challenges in CRSN and simulations are run for 11sec. Since, in [30] it is shown that energy efficiency reduces after a packet size threshold of 100bytes, frames are packeted into 100bytes chunks. Evalvid [32] is used to extract results of simulations to assess video streaming performance. For the received video, while lost packets are zero padded, during decoding lost sections are extrapolated from already reconstructed frames. 
(c)

Figure 8: Throughput (a), packet delivery(b) and energy efficiency(c) for video streaming in CRSN.

\subsubsection{Throughput, Packet Delivery and Energy Efficiency}

Throughput, packet delivery and energy efficiency results of CRSN nodes using TFRC for different power grid environments are given in Fig. 8(a), 8(b) and 8(c), respectively. Throughput and packet delivery results represent the total amount of packets sent by sources and delivered to sink, respectively. Since loss of packets means waste of energy in sensor networks, we measured energy efficiency as ratio of delivered packets to sent packets.

While the number of transmitted packets are above 650 pakcets/sec between 0 and 8 sec in Fig. 8(a), it can be seen from Fig. 8(b) that received packets at sink are always below 500packets/sec. Varying RTT due to spectrum sensing and spectrum handoff functionalities causes TFRC throughput to fluctuate frequently. Varying RTT due to spectrum sensing and spectrum handoff functionalities causes TFRC throughput to fluctuate frequently. In addition, smaller RTT values result in larger throughput values, since TFRC throughput is inversely proportional to square root of RTT [33]. TFRC throughput is inversely proportional to square root of RTT. Therefore, smaller RTT values will result in larger throughput values.

On the other hand, due to dense deployment and multi-source nature of sensor networks, while the number of injected packets into network are getting larger and larger, number of delivered packets at the sink decreases. It can be seen in Fig. 8(a) and 8(b) at first, second, third and forth seconds clearly. In addition to silent spectrum sensing periods, another reason for fluctuation of received packets at the sink is bursty nature of sensor network. After silent periods, intermediate nodes on the path to sink are exposed to high load with incoming packets. This results in drop of excessive amounts of packets. Even more, at some instants, e.g., sixth second, received packets per second drops below one third of the sent packets (throughput), e.g., while total sending rate of source nodes are 710 packets per second, receiving rate of sink node is about 220 packets per second. Moreover, more than half of the energy is wasted for all different power grid environments as given in Fig. 8(c).

(a) (b)

(c) (d)

Figure 9: End-to-end packet packet delay(a), pdf of end-to-end packet delay(b), interframe gap of received frames(c) and cumulative jitter(d) for video streaming in CRSN.

\subsubsection{Frame End-to-end Delay and Jitter}

In Fig. 9(a), (b) and (c) frame end-to-end delay measurements per frame are given for 500kV substation, main power room and underground transformer vault, respectively. Only in underground transformer vault all of the frames could be delivered to sink at the end of 11th second. However, frame end-to-end delay measured for underground transformer vault environment mostly stays above of $1 \mathrm{sec}$ and reaches over $2.5 \mathrm{sec}$ for some frames in Fig. 9(c), which is inappropriate for real-time video application considerations in sensor network. On the other hand, although more than 50 of last frames are not delivered, frames are exposed to less delay at $500 \mathrm{kV}$ substation environment, in Fig. 9(a), with respect to other environments, in Fig. 9 (b) and (c). Due to the tough spectrum environment of $500 \mathrm{kV}$ substation with respect to other power grid environments, more packets are lost on the way to sink because of wireless propagation. Although this incurs retransmission of lost packets, it resolves the bottleneck on the sink path. However, this is not an efficient 
solution to reduce end-to-end delay, since energy is wasted in this way. On the other hand, it can be seen from Fig. 8(c) most energy efficient operation is obtained in $500 \mathrm{kV}$ substation environment. Consecutive timeouts due to not arriving acknowledgement packets from sink is the main reason for rate reduction for source sensor nodes, while this leaves some of the sensor nodes out of event delivery, it helps others to deliver their packets to sink better, however, the resultant picture is still far from meeting timely delivery requirement of real-time applications. End-to-end frame delay reaches values over $3 \mathrm{sec}$ around frame index 150 for main power room environment in Fig. 9(b), afterwards it decreases up to $2 s e c$ due to timeout events and packet drops with reduction in rate. However, in addition to excessive amount of delay, around 30 of the frames could not be delivered to the sink.

Probability distribution function of delay is given in Fig. 9(b) for $500 \mathrm{kV}$ substation, main power room and underground transformer vault environments, respectively. Probability reaches its peak value around delay values frequently measured. In Fig. 9(b), for substation environment exposed end-to-end packet delay is mostly around 1sec. When compared main power room and underground network transformer vault environments, which have a exposed delay mostly around $2.7 \mathrm{sec}$ and $1.5 \mathrm{sec}$, respectively, $500 \mathrm{kV}$ substation environment is likely to sustain less delay. However, it is still far from allowing real-time communication in CRSN, and takes values up to $2.8 \mathrm{sec}$. End-to-end delay values around $2.7 \mathrm{sec}$ are more frequently observed in Fig. 9(b) for main power room environment. Additionally, in Fig. 9(b) end-to-end delay measurements around $1.5 \mathrm{sec}$ get higher probability for underground network transformer vault environment, again it is very high for real-time video streaming considerations.

The difference between frame arrivals are pointed out in Fig. 9(c) for $500 \mathrm{kV}$ substation, main power room and underground transformer vault. High inter-frame gaps cause freezing, e.g. decreases quality, of the video, and prevents real-time streaming. However, in all of three environments inter-frame gap reach to 1 sec and over. Spectrum sensing durations and spectrum handoff intervals are also important for inter-frame gap minimization. Intermittent connectivity due to licensed user activity makes it hard to satisfy application specific quality of service (QoS) requirements of CRSN. This property makes CRSN extremely challenging for real-time video streaming.

Variance of the inter-frame time is named as cumulative frame jitter. In Fig. 9, jitter values are acceptable only for the frames at beginning of the video. Then jitter starts to fluctuates and reaches $1.8,2.4$ and $1.5 \mathrm{sec}$ as maximum values for $500 \mathrm{kV}$ substation, main power room and underground transformer vault, respectively. Injection of more packets into network in sensor network increases the congestion in the network and causes bursty frame losses which boosts the difference between successful frame arrivals. Moreover, periodic spectrum sensing intervals and spectrum handoffs based on licensed user activities contribute to increase in jitter. In addition to traditional sensor network challenges, challenges introduced by CRSN, e.g., spectrum sensing durations and spectrum handoff intervals, amplifies the jitter and should be inspected deliberately to satisfy delay constraints of running application.

\subsubsection{Peak Signal to Noise Ratio (PSNR)}

Figure 10: Frame PSNR values of received video in different power grid environments.

In order to have acceptable quality, real-time video streaming should have an average PSNR more than $30 d B$ for each frame. In Fig. 10, obtained PSNR of the delivered frames are shown for different spectrum environments in power grid. Since as time goes on, i.e., as more packets are released by source nodes to be delivered at sink, network tends to get more congested, and hence, for higher frame indexes resultant PSNR is lower than previous ones. In $500 \mathrm{kV}$ substation environment lowest PSNR values for received frames are observed, i.e., $12.6 d B$. In relatively better spectrum conditions having main power room and underground network transformer vault environments PSNR values of frames start above $30 d B$, however as frame index increases PSNR values decreases to below $25 \mathrm{~dB}$, which implies received video quality is poor. 


\section{Observations and Future Research Directions}

Energy-efficiency has been the main focus of transport layer solutions proposed for sensor networks thus far [5]. For this aim, designed solutions mainly concentrated on rate control algorithms that provide reliability while keeping network away from congestion. However, with the sensor nodes equipped with cognitive radio, in addition to energy efficiency, dynamic spectrum access (DSA) should be transparent to event delivery. Additionally, many real-world applications, such as multimedia applications, have strict delay bounds and hence mandate timely transport of the event features from the sensor field to the sink node. Dynamic spectrum management also brings additional challenges to sensor network characteristics for real-time transport in CRSN. Overall, performance evaluations in Section III provide valuable insights to clarify challenges posed by DSA combined with the unique characteristics of sensor networks. In this section, the main results of the simulations are outlined and open research issues for multimedia and delay-sensitive data transport in CRSN are summarized.

\subsection{Overview of Simulation Results}

Extensive simulations address the existing challenges in the design of transport protocols, and show that transport layer performance suffers from cognitive cycle introduced with DSA. Results are outlined briefly as follows.

- Spectrum sensing, one of the main functionalities in cognitive radio, causes silent periods that nodes sense the channel. In these silent periods packet delivery to sink stops. Therefore, extra delays induced on event information carrying and feedback packets.

- Varying the $\tau_{S}^{s} / \tau_{S}^{d}$ ratio causes network change its congestion condition. While larger $\tau_{S}^{s} / \tau_{S}^{d}$ ratio allows source nodes to send less packets in a given time, in this case congestion is mainly concentrated on source nodes. However, as the $\tau_{S}^{s} / \tau_{S}^{d}$ ratio decreases more packets are injected into network, and hence, drops on the path to sink are observed until $\tau_{S}^{s} / \tau_{S}^{d}$ ratio decreases sufficiently to allow all nodes attending real-time transport from source nodes to sink as in 6(b) and 7(b).

- Accurate RTT and packet loss rate measurements gain importance in analytical rate control schemes, since extra delays and packet drops caused by cognitive radio reduces the quality of multimedia streaming (Fig. 9 and 10 ) and timeliness of delay-sensitive data (Fig. 6(c) and 7(c)).

- With the detection of licensed user activity, sensor nodes are required to leave the channel and must find a vacant channel to maintain ongoing communication. This brings disruptions on the source to sink path. Due to unawareness of licensed user detection and spectrum handoff functionality, transport layer continues to send packets. Due to this fact packet delivery ratio decreases drastically in Fig. 4(d) and $5(\mathrm{~d})$ for $P_{O N}=\frac{9}{10}$.

- Timeliness of delivered packets are critical in real-time applications. In delay-sensitive data delivery simulations (Fig. 4(c) and 5(c)) and multimedia simulations (Fig. 9), it is shown that exposed delay is very high satisfying real-time QoS requirements. Therefore, minimization of incurred delays is essential while taking resource limited nature of sensor nodes into consideration.

DSA causes to have variations in packet delivery and delay in CRSN for different spectrum characteristic. This points out the need for spectrum-aware transport layer solutions for real-time and energy efficient communication requirement for multimedia and delay-sensitive transport in CRSN. Effects of resultant extra delays and packet losses due to incorporation of cognitive radio in sensor network are investigated in simulation results, and these results show the need for DSA-aware transport protocols considering bursty many-to-one flow in CRSN. 


\subsection{Open Research Issues}

Multimedia and delay-sensitive data transport in CRSN requires consideration of inherent characteristics of sensor network and cognitive radio challenges together to enable timely energy-efficient communication in CRSN. Overall, design of novel spectrum adaptive real-time transport solutions for CRSN is needed and spectrum awareness is the key factor to increase performance of desired transport solution. Multimedia and delay-sensitive data transport in CRSN requires special attention due to varying spectrum conditions, induced delays by spectrum sensing and spectrum handoff functionalities, and sensor network's tend to congestion. In order to enlighten further research on multimedia and delay-sensitive data transport in CRSN, we point out open research issues of transport layer as follows:

- Novel transport protocols taking DSA functionalities and challenges into consideration is required to enable energy efficient and timely delivery of multimedia and delay-sensitive data to sink. Collaboration of cognitive cycle and rate control algorithm is needed. Adaptive transmission strategies regulated by the licensed user activity and cognitive radio functionalities is essential for delay-critic applications in CRSN.

- Predictive frameworks can be developed based of probabilistic behavior pattern of licensed users. As in TFRC case, analytic models can be developed including cognitive radio parameters, spectrum conditions and licensed user activity pattern in addition to sensor network characteristics.

- Designed protocols should adapt rapidly to dynamically varying network capacity due to spectrum handoff. With the union of the cognitive radio and sensor networks, network capacity problem is further amplified and estimation of it becomes extremely challenging.

- Cross layer interactions are essential with cognitive cycle in order to benefit from cognitive radio and utilize limited resources of sensor nodes efficiently. Therefore, cross-layer rate control algorithm integrating cognitive radio functionalities and rate control algorithm is necessary to real-time communication demand in CRSN.

- Based on varying licensed user activity, spectrum sensing duration and spectrum environment, novel real-time and energy efficient algorithms required to control rate of source nodes. With spectrum handoff, in addition to introduced extra delay, spectrum environment and cognitive radio parameters such as spectrum sensing and data transmission period are changes. Novel techniques should be developed to enable seamless multimedia streaming and delay-sensitive data delivery in CRSN.

\section{Conclusion}

In this paper, CRSN challenges for multimedia and delay-sensitive data transport are explored, and emerging challenges are shown by simulation experiments in different spectrum environments. Furthermore, open research issues for the realization of energy-efficient and real-time transport in CRSN are presented. Clearly, there is a need for energy-efficient and delay-sensitive rate control mechanisms which take unique challenges of real-time communication in CRSN into account. In this regard, novel CRSN transport protocols for multimedia and delay-sensitive applications must consider communication impairments due to spectrum sensing and mobility, such as excessive delays and packet losses incurred by cognitive cycle. Overall, the performance evaluations provide valuable insights about real-time transport in CRSN and guide design decisions and tradeoffs for CRSN applications in smart electric power grid. Future work includes development of adaptive and spectrum-aware transport layer solutions and investigating the impact of different heterogeneous resources, such as transmission power, network bandwidth and processing power, on overall network performance and optimal placement of these resources in the network. 


\section{Acknowledgements}

The work of A. O. Bicen and V. C. Gungor was supported by the European Union FP7 Marie Curie International Reintegration Grant (IRG) under Grant PIRG05-GA-2009-249206. The work of O. B. Akan was supported in part by the Turkish Scientific and Technical Research Council under grant \#110E249 and by the Turkish National Academy of Sciences Distinguished Young Scientist Award Program (TUBAGEBIP).

\section{References}

[1] S. Haykin, "Cognitive Radio: Brain-empowered Wireless Communications", IEEE Journal on Selected Areas in Communications (JSAC), Vol. 23, No. 2, pp. 201-220, Feb. 2005.

[2] I. F. Akyildiz, W. Y. Lee, M. C. Vuran, and S. Mohanty, "NeXt Generation/Dynamic Spectrum Access/Cognitive Radio Wireless Networks: A Survey," Computer Networks Journal (Elsevier), vol. 50, no. 13, pp. 2127-2159, Sept. 2006.

[3] O. B. Akan, O. B. Karli, and O. Ergul, "Cognitive Radio Sensor Networks," IEEE Network, vol. 23, no. 4, pp. 34-40, July-August 2009.

[4] I. F. Akyildiz, T. Melodia, and K. R. Chowdhury, "A survey on wireless multimedia sensor networks," Computer Networks Journal (Elsevier), vol. 51, no. 4, pp. 921-960, Mar. 2007.

[5] C. Wang, M. Daneshmand, B. Li and K. Sohraby, "A survey of Transport Protocols for Wireless Sensor Networks," IEEE Network, pp. 34-40, Vol. 20, No. 3, 2006.

[6] V. C. Gungor, B. Lu, G.P. Hancke, "Opportunities and Challenges of Wireless Sensor Networks in Smart Grid - A Case Study of Link Quality Assessments in Power Distribution Systems," IEEE Transactions on Industrial Electronics, vol. 57, no. 10, pp. 3557-3564, October 2010.

[7] W. Y. Lee and I. F. Akyildiz, "Optimal Spectrum Sensing Framework for Cognitive Radio Networks," IEEE Trans. on Wireless Comm., vol. 7, no. 10, pp. 3845-3857, Oct. 2008.

[8] C. Wan, S. B. Eisenman, and A.T. Campbell, "CODA: Congestion Detection and Avoidance in Sensor Networks," in Proc. ACM Sensys 2003, Los Angeles, USA, Nov. 2003.

[9] O. B. Akan and I. F. Akyildiz, "Event-to-Sink Reliable Transport in Wireless Sensor Networks," IEEE/ACM Trans. Networking, vol. 13, pp. 1003-1016, Oct. 2005.

[10] V. C. Gungor, O. B. Akan, and I. F. Akyildiz, "A Real-Time and Reliable Transport Protocol for Wireless Sensor and Actor Networks," IEEE/ACM Trans. Networking, vol. 16, no. 2, pp. 359-370, Apr. 2008.

[11] C. Y. Wan, A. T. Campbell, and L. Krishnamurthy, "PSFQ: A Reliable Transport Protocol for Wireless Sensor Networks," in Proc. ACM WSNA 2002, pp. 1-11, Sept. 2002.

[12] F. Stan and J. Heidemann, "RMST: Reliable Data Transport in Sensor Networks," in Proc. IEEE SNPA 2003, pp. 102-112, May 2003.

[13] K. R. Chowdhury, M. Di Felice, and I. F. Akyildiz, "TP-CRAHN: A Transport Protocol for Cognitive Radio Ad-Hoc Networks," in Proc. IEEE INFOCOM 2009, Apr. 2009.

[14] S. Floyd, M. Handley, J. Padhye, and J. Widmer, "Equation-based Congestion control for unicast applications," in Proc. ACM SIGCOMM 2000, pp. 45-58, Aug. 2000.

[15] S. Floyd and K. Fall, "Promoting the Use of End-to-End Congestion Control in the Internet," IEEE/ACM Trans. Networking, vol. 7, no. 4, pp. 458-472, Aug. 1999.

[16] W. Tan and A. Zakhor, "Real-Time Internet Video Using Error Resilient Scalable Compression and TCP-Friendly Transport Protocol," IEEE Trans. Multimedia, vol. 1, no. 2, pp. 172-86, Jun. 1999.

[17] M. Chen and A. Zakhor, "Rate Control for Streaming Video over Wireless," in Proc. INFOCOM 2004, Hongkong, China, Mar. 2004.

[18] K. Sriram and W. Whitt, "Characterizing Superposition Arrival Processes in Packet Multiplexers for Voice and Data," IEEE JSAC, vol. 4, no. 6, pp. 833-846, Sept. 1986.

[19] Y. Chen, Q. Zhao, and A. Swami, "Joint Design and Separation Principle for Opportunistic Spectrum Access," in Proc. IEEE Asilomar Conference on Signals, Systems and Computers 2006, Oct. 2006.

[20] C. Chou, S. Shankar, H. Kim, and K. G. Shin, "What and How much to Gain by Spectrum Agility?", IEEE JSAC, vol. 25, no. 3, pp. 576-588, Apr. 2007.

[21] D. R. Cox, "Renewal Theory." NY: John Wiley \& Sons Inc, 1962.

[22] S. M. Amin and B. F. Wollenberg, "Toward a Smart Grid," IEEE Power and Energy Magazine, vol. 3, no. 5, pp. 34-41, Sep./Oct. 2005.

[23] A. Bose, "Smart Transmission Grid Applications and their Supporting Infrastructure," IEEE Transactions on Smart Grid, vol. 1, no. 1, pp. 11-19, June 2010.

[24] B. Heile, "Smart Grids for Green Communications," IEEE Wireless Communications, vol. 17, no. 3, pp. 4-6, June 2010.

25] V.C. Gungor, and F. C. Lambert, "A Survey on Communication Networks for Electric System Automation," Computer Netwroks Journal (Elsevier), vol. 50, pp. 877-897, May 2006.

[26] C. H. Hauser, D. E. Bakken, and A. Bose, "A Failure to Communicate: Next Generation Communication Requirements, Technologies, and Architecture for the Electric Power Grid," IEEE Power and Energy Magazine, vol.3, no.2, pp. 47- 55, March-April 2005.

[27] U.S. Department of Energy, "The Smart Grid: An Introduction," Washington, DC, Sep. 2008. 
[28] K. Moslehi, R. Kumar, "A Reliability Perspective of the Smart Grid," IEEE Transactions on Smart Grid, vol. 1, no. 1, pp. 57-64, June 2010.

[29] The ns-3 Network Simulator, http://www.nsnam.org.

[30] Y. Sankarasubramaniam, I. F. Akyildiz, and S. W. McLaughlin, "Energy Efficiency Based Packet Size Optimization in Wireless Sensor Networks," in Proc. IEEE SNPA 2003, pp. 1-8, 2003.

[31] M. C. Vuran, O. B. Akan, and I. F. Akyildiz, "Spatio-temporal Correlation: Theory and Applications for Wireless Sensor Networks," Comput. Netw. J., vol. 45, no. 3, pp. 245-261, Jun. 2004.

[32] EvalVid - A Video Quality Evaluation Tool-set, http://www.tkn.tu-berlin.de/research/evalvid.

[33] S. Floyd, M. Handley, J. Padhye, and J. Widmer, "TCP Friendly Rate Control (TFRC): Protocol Specification," in IETF RFC 5348, Sept. 2008. 